\title{
Molar Refraction, Thermal Expansivity and Polarizabilities of Aqueous 1-1 Electrolyte Solutions
}

\author{
RUPALI SETHI $^{1 *}$, N.K.SONI ${ }^{2}$, TANU SRIVASTAVA ${ }^{1}$ and \\ SURENDRA KUMAR VISHWAKARMA ${ }^{3}$ \\ ${ }^{1}$ Department of Chemistry, University of Allahabad, Allahabad, India \\ ${ }^{2}$ Department of Chemistry, Govt. P.G. College Charkhari U.P., India \\ ${ }^{3}$ Department of Chemistry, Mahatma Gandhi Chitrakoot Gramodaya Vishvavidyalaya \\ Chitrakoot, Satna, India \\ sethirupali.au@gmail.com
}

Received 26 August 2017 / Accepted 9 September 2017

\begin{abstract}
The refractive index and density of aqueous solutions of $\mathrm{LiCl}, \mathrm{NaCl}$ and $\mathrm{KCl}$ have been measured at four different temperatures $(298.15,303.15,313.15$ and $318.15 \mathrm{~K})$ in the concentration range of 0.01 to 0.1 molar. From the experimental values of density and refractive index molar refractions of electrolyte solution and electrolyte have been calculated. These data were also utilized to obtain the values of thermal expansion coefficient and polarizability using the relevant equations. The results of investigation were found to be satisfactory.
\end{abstract}

Keywords: Refractive index, Thermal expansion coefficient, Polarizability, Density

\section{Introduction}

Considerable work has been done during recent past on the refractive index and related properties of non-electrolyte solutions. This property can be used to study the molecular interactions and also can be employed to correlate important properties e.g. molar refraction, polarizability and thermal expansion coefficient. Various mixing rules ${ }^{1-7}$ for refractive index have been proposed from time to time. These mixing rules have been applied and tested by a number of workers for binary and higher order liquid mixtures. However, such study of refractive index has been quite limited for electrolyte solutions, Lee et al., ${ }^{8}$ measured refractive index of $\mathrm{LiBr}+\mathrm{H}_{2} \mathrm{O}+1,3$ propane diol system. Knowledge of refractive index $(n)$, molar refraction $\left(\mathrm{R}_{\mathrm{m}}\right)$, thermal expansion coefficient $(\alpha)$ and polarizabilities $\left(\alpha_{\mathrm{p}}\right)$ is useful for predicting many physicochemical properties of electrolyte solutions. There are several correlations between refractive index and some thermophysical properties like critical constants, density, polarizability, equation of state parameters and dielectric permittivity that indicate the importance of this property for the study of electrolytic solutions while a wealth of studies on water and electrolyte solutions by light absorption and 
scattering experiments have been reported, we pay attention here to study refractive index of aqueous solutions of electrolytes. The polarizabilities of free or gaseous ions are not susceptible to direct experimental determinations ${ }^{9}$. The purpose of the present work is to deduce thermal expansion coefficient, molar refraction and polarizibilities of ions of aqueous electrolyte solutions from the experimental data of density and refractive index. We have employed common mixing rules, Lorentz-Lorenz ${ }^{5,6}$ and Eykman (Eyk) ${ }^{7}$, for the calculation of thermal expansion coefficient $(\alpha)$ of solutions.

\section{Experimental}

Densities of solutions were measured with a single stem Pycnometer which was calibrated by using triple distilled water at $298.15 \mathrm{~K}$. The overall experimental uncertainty in the density measurement was estimated to be $\pm 0.0001 \mathrm{~g} / \mathrm{cc}$. Ultrasonic speeds of pure liquids and their solutions were measured using an ultrasonic interferometer supplied by M/S Mittal Enterprise, New Delhi, operating at a frequency of $2 \mathrm{MHz}$. The ultrasonic speeds were measured in pure liquid water, 1-butanol, 2-butanol, 2-methyl-1-propanol and 2-methyl-2 propanol at $298.15 \mathrm{~K}$. These values were compared to the literature values. The maximum error in the speed of sound relative to water at $298.15 \mathrm{~K}$ was found to be less than $0.2 \mathrm{~ms}^{-1}$. The refractive index of pure water and electrolytic solutions were measured directly with calibrated and thermostatic instrument called Abbe Refractometer at wavelength of $D$ sodium line $589.3 \mathrm{~nm}$. The instrument was calibrated by using the aforesaid liquids at $298.15 \mathrm{~K}$. A thermostatically controlled bath was used for temperature measurements giving precision of $+0.1 \mathrm{~K}$. The electrolytes $\mathrm{LiCl}, \mathrm{NaCl}$ and $\mathrm{KCl}$ used were of A.R. grade. The solutions were proposed in triple distilled water. The details of experimental procedure for measuring density, refractive index and ultrasonic speed are described in earlier papers ${ }^{7,10}$.

\section{Formulation}

Density, refractive index and ultrasonic speed data of aqueous $\mathrm{LiCl}, \mathrm{NaCl}$ and $\mathrm{KCl}$ solutions were employed to obtain important and useful properties with the help of relations outlined here.

$$
\text { Molar refraction, } R_{m}=\frac{n^{2-1}}{n^{2+2}} . V m
$$

$V_{m}$ is the molar volume of electrolytic solution $R_{m}$ of solute electrolyte can be calculated by the relation,

Hence,

$$
\mathrm{R}_{\mathrm{m} \text {,(soln.) }}=\mathrm{R}_{\mathrm{m} \text {,(solvent) }}+\mathrm{R}_{\mathrm{m} \text {,(solute) }}
$$

$$
\mathrm{R}_{\mathrm{m} \text { (electrolyte) }}=\mathrm{R}_{\mathrm{m} \text {,(soln.) }}-\mathrm{R}_{\mathrm{m} \text {, (water) }}
$$

$\mathrm{R}_{\mathrm{m} \text { (soln) }}$ and $\mathrm{R}_{\mathrm{m} \text { (water) }}$ are given by

$$
R_{m(s o \mathrm{ln} .)}=\frac{n_{s o \mathrm{ln} .}^{2}-1}{n_{s o \mathrm{ln} .}^{2}+2} \cdot \frac{\sum M i X i}{\rho_{s o \mathrm{ln} .}}
$$

Where

$$
\begin{gathered}
\frac{\sum M i X i}{\rho_{\text {soln. }}}=\frac{M_{\text {salt }} X+M_{w} X_{w}}{\rho_{\text {soln }} .} \\
R_{m(w)}=\frac{n_{w}^{2}-1}{n_{w}^{2}+2} \cdot \frac{M w}{\rho w}
\end{gathered}
$$




$$
R_{m(\text { salt })}=\frac{n_{\text {salt }}^{2}-1}{n_{\text {salt }}^{2}+2} \cdot \frac{M_{\text {salt }}}{\rho_{\text {salt }}}
$$

The polarizability, $\alpha_{\mathrm{p}}$, of the solution is obtained from equation,

$$
\alpha_{p}=\frac{3 R_{m}}{4 \pi N}
$$

Where $\mathrm{N}$ is the Avogadro number, using the temperature dependant data of density, the values of thermal expansion coefficient $(\alpha)$ of solution is obtained from the relation.

$$
\alpha=\rho\left(\frac{\partial_{\rho-1}}{\partial T}\right)_{P}
$$

The temperature dependent refractive index data has been employed to calculate $\alpha$ of binary electrolyte solution. This approach has been recently employed ${ }^{11-13}$ for binary liquid mixtures. Lorentz-Lorenz (L-L) ${ }^{4,5}$ and Eykman (Eyk) ${ }^{14}$ mixing rules for refractive index were employed to obtain $\alpha$ using the following equation;

$$
\begin{gathered}
\alpha_{L-L} \frac{-6 n}{\left(n^{2}-1\right)\left(n^{2}+2\right)}\left(\frac{d n}{d t}\right) \\
\alpha_{E y k}=-\frac{\left(n^{2}+0.8 n+1\right)}{\left(n^{2}-1\right)(n+0.4)}\left(\frac{d n}{d t}\right)
\end{gathered}
$$

\section{Results and Discussion}

Experimental values of density and refractive index of aqueous solutions of $\mathrm{LiCl}, \mathrm{NaCl}$ and $\mathrm{KCl}$ have been used to calculate molar refraction, polarizability and thermal expansivity at five different temperatures $298.15 \mathrm{~K}, 303.15 \mathrm{~K}, 308.15 \mathrm{~K}, 313.15 \mathrm{~K}$ and $318.15 \mathrm{~K}$ using various relations. The calculated values of $R_{m}, \alpha_{p}, \alpha$ are reported in Tables 1-3. A perusal of Tables 1-3 reveals that the polarizability, thermal expansivity and molar refraction values of solution and solvent decrease with increasing temperature for all the binary electrolytic solutions over the entire concentration range. The values of all the thermodynamic properties also increase with increasing concentration of the solution. The trends of increasing all the properties are the same in all the solutions. Thermal expansion coefficient of electrolyte solution has been determined by using Eqs 8-10. This property is linked with the electrostriction of water in the vicinity of ions and changes in the water structure (hydrogen-bonding equilibrium among water molecules). The decrease in thermal expansivity by raising the temperature is due to the ion-water interactions. Molar refraction has been calculated using Eq. 1.The calculated molar refractions are included in Table 1, the molar refraction values of the electrolyte solutions assuming the rule of mixtures holds, are recorded. In the case of electrolyte solutions only positive values of $R_{m}$ are observed. $R_{m}$ values employed to explain interaction effects in electrolytic solutions. From the above, it can be calculated that interactions depend on the concentration and temperature. The polarizability of a compound is a measure of its molecular volume. In the phenomenon of refraction of light it is the loosely bound (called active) electrons of the ions which play a prominent role. Since the electrons of the cation are very firmly held owing to the positive charge and the opposite holds in the case of the anion, it is the latter ion which makes the principal contribution to the molar refraction. 
Table 1. Molar refraction of aqueous solutions and electrolyte $\left(\mathrm{R}_{\mathrm{m}, \text { elec }}\right)$ of $\mathrm{LiCl}, \mathrm{NaCl}$ and $\mathrm{KCl}$, as a function of concentration and temperature

\begin{tabular}{|c|c|c|c|c|c|c|c|c|c|}
\hline \multicolumn{10}{|c|}{$\mathrm{LiCl}$} \\
\hline \multirow{2}{*}{$\begin{array}{l}\text { Conc., } \\
\mathrm{mL}^{-1}\end{array}$} & \multicolumn{2}{|c|}{$298.15 \mathrm{~K}$} & \multicolumn{2}{|c|}{$303.15 \mathrm{~K}$} & $308.15 \mathrm{~K}$ & \multicolumn{2}{|c|}{$313.15 \mathrm{~K}$} & \multicolumn{2}{|c|}{$318.15 \mathrm{~K}$} \\
\hline & Soln. & Elec. & Soln. & Elec. & Soln. & Soln. & Elec. & Soln. & Elec. \\
\hline 0.1 & 10.0659 & 6.4267 & 10.0613 & 6.4315 & 10.05036 .4223 & 0.0251 & 16.3973 & 9.9714 & 6.3386 \\
\hline 0.09 & 10.0623 & 423 & 5 & ( & 10.03 & 68 & 6.389 & 9.9811 & 6.34 \\
\hline 0.08 & 7 & 6 & 1 & 6 & 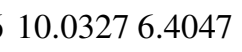 & & 6.3 & 782 & 3 \\
\hline 0.07 & 10 & 6 & 1 & 6 & 1 & & 09 & 9.9924 & 0.53 \\
\hline 0.06 & 10.0523 & 6.413 & .0478 & 6.418 & 10.04 & & 96.3961 & 10.01 & 6.37 \\
\hline 0.05 & 5 & 4132 & 1 & 6.42 & 10.0 & 3 & 36.3 & 10.0211 & 6.38 \\
\hline 0.04 & 7 & +194 & 36 & 6.4238 & 10.0 & 9 & 96.4001 & 10.0334 & 6.4006 \\
\hline 0.03 & 10.0639 & 6.4246 & 10.0629 & 6.4331 & 10.04 & & 6.4083 & 46 & 6.4119 \\
\hline 0.02 & 3 & 426 & 1 & 6 & 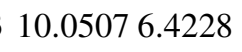 & & 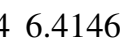 & & 6.4223 \\
\hline 0 & & 6 & & ( & $\begin{array}{c}10.0562 \\
\mathrm{NaCl}\end{array}$ & & & & 6.431 \\
\hline 0.1 & & 6 & & 4 & 10 & & & UJ & $2 J$ \\
\hline 0.09 & 2 & 799 & 33 & 6.4635 & 10. & .0504 & 26 & 5 & 0.4 \\
\hline 0.08 & 0.1 & 6.4671 & 083 & 6.4532 & $10.074 \quad 6.446$ & 68 & 6.419 & 10 & 6.4645 \\
\hline 0.07 & 7 & 6. & 10 & 4 & 10 & 1 & 10 & & ( \\
\hline 0 & 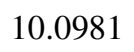 & 6 & 2 & 2 & $10.077 \quad 6.449$ & 1 & 7 & 10 & 6.4697 \\
\hline 0.05 & 27 & 6.4534 & & 6.426 & 10.06 & & 6.4361 & 047 & 6.472 \\
\hline 0.04 & 0 & 6.4567 & 10.0555 & 6.4256 & 10 & 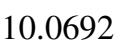 & 4 & 59 & 6.4742 \\
\hline 0 & & & & 6 & 1 & & & & \\
\hline 0.02 & 1 & 662 & & 6 & 10 & & 6.439 & 91 & 6.4569 \\
\hline 0.01 & 1 & 17 & 5 & 2 & $\begin{array}{c}10.06256 .4 \\
\mathrm{KCl}\end{array}$ & 67 & 89 & 10.077 & 6.4 \\
\hline 0 & & & & 6.42 & 6.3816 & 75 & 6.3697 & 9.9904 & 6.3577 \\
\hline 0 & 7 & $t$ & & & 1 & S & 6.366 & 10 & 0 \\
\hline 0.08 & & 8 & & 7 & 10 & 92 & 6.3 & 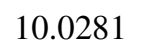 & 53 \\
\hline 0.07 & 10 & 079 & & 6.3941 & 10.00056 .3725 & 9.9874 & 6.3596 & 93 & 6.3965 \\
\hline 0.06 & 10 & 5 & 10 & 6.3922 & 9.99976 .37 & 55 & 6.3 & 10 & 6.3961 \\
\hline $00 \tau$ & 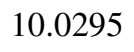 & 2 & 5 & 6.3848 & 9.99896 .3709 & 9.9953 & 6.3675 & 10. & 6.35 \\
\hline 0.04 & 231 & 838 & 10.0198 & 6.39 & 10.01016 .3821 & 10.0081 & 6.3803 & 10.0304 & 6.3976 \\
\hline 0.03 & 10 & 6. & 03 & 2 & 10 & 5 & 67 & 10. & 6.3998 \\
\hline 000 & 10. & 6 & 10.0342 & 6 & 10. & 100187 & 9 & 10. & ( \\
\hline 0.01 & 10.034 & 6.3948 & 10.0366 & 6.4068 & 10.03076 .4027 & 10.0302 & 6.4024 & 10.0453 & 6.4126 \\
\hline
\end{tabular}


Table 2. Experimental and calculated values (from " Eq. 10 and ${ }^{\#} \mathrm{Eq} .11$ ) of thermal expansion coefficient of aqueous solutions of $\mathrm{LiCl}, \mathrm{NaCl}, \mathrm{KCl}$, as a function of concentration and temperature

\begin{tabular}{|c|c|c|c|c|c|c|c|c|c|c|c|c|c|c|c|}
\hline \multirow{3}{*}{$\begin{array}{c}\mathrm{C} \\
\mathrm{mol} \\
/ \mathrm{Lit}\end{array}$} & \multicolumn{15}{|c|}{$\mathrm{LiCl}$} \\
\hline & \multicolumn{3}{|c|}{$298.15 \mathrm{~K}$} & \multicolumn{3}{|c|}{$303.15 \mathrm{~K}$} & \multicolumn{3}{|c|}{$308.15 \mathrm{~K}$} & \multicolumn{3}{|c|}{$313.15 \mathrm{~K}$} & \multicolumn{3}{|c|}{$318.15 \mathrm{~K}$} \\
\hline & Exp. & Cal." & Cal. $^{\#}$ & Exp. & Cal." & Cal. $^{\#}$ & Exp. & Cal." & Cal. $^{\#}$ & Exp. & Cal." & Cal. $^{\#}$ & Exp. & Cal. & Cal. $^{\#}$ \\
\hline 0.1 & 3.0975 & 4.3066 & .5007 & 3.0978 & 4.3096 & 4.5036 & 3.0988 & 4.317 & 4.5108 & 3.0997 & 4.332 & 4.5252 & 3.1143 & 4.3486 & 4.5411 \\
\hline 0.09 & 2.9982 & 4.3096 & 4.5036 & 2.9985 & 4.311 & 4.505 & 2.9993 & 4.3245 & 4.518 & 3.0006 & 4.338 & 4.531 & 3.0181 & 4.3546 & 4.547 \\
\hline 0.08 & 2.8986 & 4.3125 & 4.5065 & 2.8993 & 4.314 & 4.5079 & 2.9003 & 4.329 & 4.5223 & 2.9012 & 4.3425 & 4.5353 & 2.9155 & 4.3562 & 4.5484 \\
\hline 0.07 & 3.0191 & 4.2034 & 4.3922 & 3.0197 & 4.2049 & 4.3936 & 3.0236 & 4.2209 & 4.4091 & 3.0254 & 4.2327 & 4.4204 & 3.0391 & 4.2445 & 4.4317 \\
\hline 0.06 & 3.6793 & 4.0927 & 4.2764 & 3.6807 & 4.097 & 4.2805 & 3.6874 & 4.1098 & 4.2928 & 3.6896 & 4.1212 & 4.3038 & 3.7088 & 4.1327 & 4.3149 \\
\hline 0.05 & 3.8804 & 4.2626 & 4.4538 & 3.8843 & 4.267 & 4.458 & 3.8905 & 4.2803 & 4.4709 & 3.8936 & 4.2938 & 4.4838 & 3.9137 & 4.3057 & 4.4953 \\
\hline 0.04 & 3.8639 & 4.2641 & 4.4552 & 3.8673 & 4.2715 & 4.4623 & 3.8732 & 4.2833 & 4.4737 & 3.8771 & 4.2982 & 4.4881 & 3.8966 & 4.3072 & 4.4967 \\
\hline 0.03 & 97 & 4.2655 & 4.4566 & 3 & 4.2729 & 4.4637 & & 4.2863 & 4.4766 & & 4.2997 & 4.4895 & 98 & 4.3087 & 4.4982 \\
\hline 0.02 & 3.9699 & 4.2685 & 4.4595 & 3.97 & 4.2744 & 4.4652 & 87 & 4.2878 & 78 & & 4.3012 & 91 & & 4.3118 & 4.5011 \\
\hline 0.01 & 4.2331 & 4.2715 & 4.4623 & 4.2387 & 4.2759 & 4.4666 & & 4.2908 & 4.4809 & 4.2 & 4.3027 & 4.4924 & 4.2713 & 4.3163 & 4.5054 \\
\hline 0.1 & 2967 & 5.0462 & 5.2773 & 3 & 5.0548 & 57 & 3.2984 & 5.0793 & 091 & 99 & 986 & 77 & 1 & 5.1039 & 5.3328 \\
\hline 0.09 & 3.0975 & 4.2786 & 4.4739 & 3.0981 & 4.2934 & 4.4881 & 3.0 & 4.3008 & 4.4952 & 97 & 4. & 4. & 163 & 232 & 4.5167 \\
\hline 0.08 & 079 & 3.8407 & 4.0154 & 3.0085 & 3.8526 & 4.0269 & & 3.8593 & 333 & 03 & 727 & 461 & & 3.8767 & 0500 \\
\hline 0.07 & 3.0182 & 3.5648 & 3.7268 & 3.0191 & 3.5784 & 3.7398 & 3.023 & 3.5821 & 3.7434 & 3.0248 & 3.5933 & 3.7541 & 382 & 3.597 & 3.7577 \\
\hline 0.06 & 3.4586 & 3.5116 & 3.6709 & 3.4593 & 3.5237 & 3.6826 & 3.4659 & 3.5298 & 3.6884 & 3.468 & 3.5396 & 3.6978 & 844 & 3.542 & 3.7002 \\
\hline 0.05 & 3.4797 & 2.8456 & 2.9745 & 3.4835 & 2.8624 & 2.9906 & 3.4891 & 2.8664 & 2.9944 & 4912 & 674 & 2.9954 & 3.5063 & 2.8684 & 2.9964 \\
\hline 0.04 & 17 & 2.7348 & 2.8586 & 52 & 2.7528 & 2.8759 & 31 & 2.7548 & 2.8778 & 37 & 2.7557 & 2. & 3.4476 & 2.7567 & 2.8796 \\
\hline 0.03 & 3.4245 & 2.7918 & 2.918 & 3.4303 & 2.8111 & 2.9367 & 3.4334 & 2.8121 & 2.9376 & 3.4382 & 2.8131 & 2.9385 & 3.45 & 2.815 & 2.9404 \\
\hline 0.02 & 3.3467 & 3.9097 & 4.0864 & 3.3517 & 3.9382 & 4.1138 & 3.3551 & 3.9423 & 4.1177 & 3.3602 & 3.9477 & 4.123 & 3.3707 & 3.9519 & 4.1270 \\
\hline 0.01 & 3.6101 & 3.9205 & 4.0968 & 3.6 & 3.9436 & 4. & $\begin{array}{c}3.6192 \\
\mathrm{KCl}\end{array}$ & 3.9464 & 4. & 68 & 546 & 4.1296 & 67 & 3.9629 & 4.1376 \\
\hline 0.1 & 964 & 1778 & 3666 & 7 & 08 & 91 & 2.9976 & 4.2126 & 01 & & 4.2199 & 72 & 03 & 4.2273 & 4.4142 \\
\hline 0.09 & 2.9967 & 3.8085 & 3.9797 & 2.9976 & 3.8203 & 3.9911 & 2.9979 & 3.8336 & 4.0039 & 2.9988 & 3.839 & 4.0091 & 3.0114 & 3.8443 & 4.0142 \\
\hline 0.08 & 2.9973 & 3.8111 & 3.9823 & 2.9982 & 3.8217 & 3.9924 & 2.9985 & 3.8349 & 4.0052 & 2.9991 & 3.8403 & 4.0103 & 3.0142 & 3.847 & 4.0168 \\
\hline 0.07 & 2.9979 & 3.8124 & 3.9835 & 2.9985 & 3.8243 & 3.995 & 91 & 3.8363 & 4.0065 & 2.9994 & 3.843 & 4.0129 & 3.0154 & 3.8483 & 4.0181 \\
\hline 0.06 & 2.9982 & 3.8725 & 4.046 & 2.9988 & 3.8819 & 4.055 & 2.9997 & 3.8941 & 4.0667 & 3.0006 & 3.8927 & 4.0654 & 3.0178 & 3.909 & 4.0811 \\
\hline 0.05 & 2.9985 & 3.6531 & 3.8163 & 2.9991 & 3.6607 & 3.8236 & 3.0003 & 3.6696 & 3.8322 & 3.006 & 3.6798 & 3.842 & 3.0187 & 3.685 & 3.8470 \\
\hline 0.04 & 2.9991 & 3.3193 & 3.4673 & 3.0015 & 3.3239 & 3.4717 & 3.0045 & 3.332 & 3.4795 & 3.009 & 3.339 & 3.4862 & 3.0208 & 3.346 & 3.4930 \\
\hline 0.03 & 2.9997 & 3.2653 & 3.4107 & 3.0054 & 3.2687 & 3.414 & 3.0066 & 3.2767 & 3.4217 & 3.0127 & 3.2847 & 3.4294 & 3.0224 & 3.2904 & 3.4349 \\
\hline 0.02 & 3.0006 & 3.1549 & 3.2952 & 3.0075 & 3.1571 & 3.2973 & 3.0099 & 3.1648 & 3.3047 & 3.0148 & 3.1725 & 3.3122 & 3.0257 & 3.178 & 3.3175 \\
\hline 0.01 & 3.0066 & 3.2687 & 3.414 & 3.0099 & 3.2721 & 3.4173 & 3.0133 & 3.2789 & 3.4238 & 3.0199 & 3.2881 & 3.4327 & 3.0288 & 3.2938 & 3.4382 \\
\hline
\end{tabular}


Table 3. Polarizability of aqueous solution and electrolytes of $\mathrm{LiCl}, \mathrm{NaCl}$, and $\mathrm{KCl}$ as a function of concentration and temperature

\begin{tabular}{|c|c|c|c|c|c|c|c|c|c|c|}
\hline \multirow{3}{*}{$\begin{array}{l}\text { Conc., } \\
\text { mol/Lit }\end{array}$} & \multicolumn{10}{|c|}{$\mathrm{LiCl}$} \\
\hline & \multicolumn{2}{|c|}{$298.15 \mathrm{~K}$} & \multicolumn{2}{|c|}{$303.15 \mathrm{~K}$} & \multicolumn{2}{|c|}{$308.15 \mathrm{~K}$} & \multicolumn{2}{|c|}{$313.15 \mathrm{~K}$} & \multicolumn{2}{|c|}{$318.15 \mathrm{~K}$} \\
\hline & Soln. & Elec. & Soln. & Elec. & Soln. & Elec. & Soln. & Elec. & Soln. & Elec. \\
\hline 0.1 & 3.992 & 2.549 & 3.99 & 2.551 & 3.986 & 2.547 & 3.976 & 2.537 & 3.954 & 2.514 \\
\hline 0.09 & 3.99 & 2.547 & 3.99 & 2.55 & 3.981 & 2.542 & 3.972 & 2.534 & 3.958 & 2.518 \\
\hline 0.08 & 3.989 & 2.545 & 3.988 & 2.549 & 3.979 & 2.54 & 3.97 & 2.531 & 3.957 & 2.516 \\
\hline 0.07 & 3.987 & 2.544 & 3.987 & 2.547 & 3.98 & 2.541 & 3.973 & 2.534 & 3.963 & 2.522 \\
\hline 0.06 & 3.986 & 2.543 & 3.985 & 2.545 & 3.982 & 2.543 & 3.975 & 2.536 & 3.97 & 2.53 \\
\hline 0.05 & 3.986 & 2.543 & 3.987 & 2.548 & 3.983 & 2.545 & 3.977 & 2.538 & 3.974 & 2.533 \\
\hline 0.04 & 3.989 & 2.546 & 3.987 & 2.547 & 3.984 & 2.545 & 3.977 & 2.538 & 3.979 & 2.538 \\
\hline 0.03 & 3.991 & 2.548 & 3.991 & 2.551 & 3.984 & 2.545 & 3.98 & 2.541 & 3.983 & 2.543 \\
\hline 0.02 & 3.992 & 2.548 & 3.992 & 2.552 & 3.986 & 2.547 & 3.982 & 2.544 & 3.988 & 2.547 \\
\hline 0.01 & 3.992 & 2.549 & 3.994 & $\begin{array}{l}2.554 \\
\mathrm{NaCl}\end{array}$ & 3.988 & 2.549 & 3.987 & 2.548 & 3.991 & 2.55 \\
\hline 0.1 & 4.019 & 2.576 & 4.014 & 2.574 & 4 & 2.561 & 3.988 & 2.55 & 3.999 & 2.559 \\
\hline 0.09 & 4.013 & 2.57 & 4.003 & 2.563 & 3.998 & 2.56 & 3.986 & 2.547 & 4.004 & 2.563 \\
\hline 0.08 & 4.008 & 2.565 & 3.999 & 2.559 & 3.995 & 2.556 & 3.984 & 2.546 & 4.004 & 2.564 \\
\hline 0.07 & 4.006 & 2.563 & 3.995 & 2.555 & 3.997 & 2.558 & 3.989 & 2.55 & 4.003 & 2.563 \\
\hline 0.06 & 4.005 & 2.561 & 3.994 & 2.555 & 3.996 & 2.557 & 3.99 & 2.551 & 4.006 & 2.566 \\
\hline 0.05 & 4.002 & 2.559 & 3.988 & 2.548 & 3.99 & 2.551 & 3.991 & 2.552 & 4.007 & 2.567 \\
\hline 0.04 & 4.004 & 2.561 & 3.988 & 2.548 & 3.991 & 2.552 & 3.993 & 2.554 & 4.008 & 2.567 \\
\hline 0.03 & 4.006 & 2.563 & 3.99 & 2.551 & 3.993 & 2.554 & 3.997 & 2.559 & 4.009 & 2.568 \\
\hline 0.02 & 4.008 & 2.564 & 3.99 & 2.551 & 3.991 & 2.552 & 3.992 & 2.554 & 4.001 & 2.561 \\
\hline 0.01 & 4.002 & 2.559 & 3.988 & $\begin{array}{c}2.548 \\
\mathrm{KCl}\end{array}$ & 3.99 & 2.552 & 3.992 & 2.553 & 3.996 & 2.556 \\
\hline 0.1 & 3.995 & 2.551 & 3.985 & 2.546 & 3.97 & 2.531 & 3.965 & 2.526 & 3.962 & 2.521 \\
\hline 0.09 & 3.986 & 2.543 & 3.977 & 2.538 & 3.967 & 2.528 & 3.963 & 2.525 & 3.975 & 2.535 \\
\hline 0.08 & 3.985 & 2.542 & 3.977 & 2.538 & 3.966 & 2.527 & 3.963 & 2.524 & 3.977 & 2.536 \\
\hline 0.07 & 3.984 & 2.541 & 3.975 & 2.536 & 3.966 & 2.527 & 3.961 & 2.522 & 3.977 & 2.537 \\
\hline 0.06 & 3.981 & 2.538 & 3.974 & 2.535 & 3.966 & 2.527 & 3.968 & 2.529 & 3.977 & 2.536 \\
\hline 0.05 & 3.977 & 2.534 & 3.971 & 2.532 & 3.965 & 2.526 & 3.964 & 2.525 & 3.976 & 2.535 \\
\hline 0.04 & 3.975 & 2.532 & 3.974 & 2.534 & 3.97 & 2.531 & 3.969 & 2.53 & 3.978 & 2.537 \\
\hline 0.03 & 3.973 & 2.53 & 3.978 & 2.538 & 3.971 & 2.533 & 3.971 & 2.533 & 3.979 & 2.538 \\
\hline 0.02 & 3.972 & 2.529 & 3.979 & 2.54 & 3.975 & 2.536 & 3.973 & 2.534 & 3.982 & 2.541 \\
\hline 0.01 & 3.979 & 2.536 & 3.98 & 2.541 & 3.978 & 2.539 & 3.978 & 2.539 & 3.984 & 2.543 \\
\hline
\end{tabular}

\section{Conclusion}

Density and refractive index of aqueous solutions of four 1-1 electrolytes have been accurately measured at $298.15,303.15,313.15$ and $318.15 \mathrm{~K}$. These data were employed to obtain molar refraction and polarizability of solutions. The results are discussed in terms of ion-solvent interactions. 


\section{References}

1. Heller W, J Phys Chem., 1965, 69(4), 1123-1129; DOI:10.1021/j100888a006

2. Dale D and Gladstone F, Phil Trans., 1858, 148, 887-894; 1863, 153, 317; DOI:10.1098/rstl.1858.0002

3. Wiener O, Zur Theorie der Refraktionskonstanten Ber. Verh. Koniglich Sachisischen Ges. Wiss. Leipzig Math Phys., 1910, 62, 256-277.

4. Lorenz L V, Ann der Phys., 1880, 11, 70.

5. Lorentz H A, Ann Phys., 1880, 245(9), 641-655.

6. Aralaguppi M I, Aminabhavi T M, Ramachandra H B and Harogoppad S B, J Chem Eng Data, 1992, 37(3), 298-303; DOI:10.1021/je00007a006

7. Pandey J D, Rai R D, Shukla R K, Tiwari K P and Shukla A K, Ind J Pure Appl Phys., 1992, 30, 94-96.

8. Lee J W, Kim J S, Lee Y W, Baek Y S and Pang H S, J Chem Thermodyn., 2001, 33(11), 1527-1534; DOI:10.1006/jcht.2001.0866

9. Pauling L, Proc R Soc London, Ser A, 1927, 114, 181-211.

10. Pandey J D, Jain P, Vyas V, Can J Chem., 1994, 72(12), 2486-2492; DOI:10.1139/v94-315

11. Pal A and Kumar H, J Chem Thermodyn., 2004, 36(3), 173-182; DOI:10.1016/j.jct.2003.11.003

12. Hosoda H, Mori H, Sogoshi N, Nagasawa A and Nakabayashi S, J Phys Chem., 2004, 108(9), 1461-1464; DOI:10.1021/jp0310145

13. Salanne M, Vuilleumier, Madded PA, Simon C, Turq P and Guillot B, J Phys Condens Matter., 2008, 20, 494207; DOI:10.1088/0953-8984/20/49/494207

14. Giner B, Lafuente C, Villares A, Haro M and Lopez M C, J Chem Thermodyn., 2007, 39(1), 148-157; DOI:10.1016/j.jct.2006.05.003 